Submitted to

Plasma Sources: Sci. Tech.

\title{
Optical Emission Spectra of a Copper Plasma Produced by a Metal Vapor Vacuum Arc Plasma Source
}

\author{
B. Yotsombat ${ }^{1}$, S. Davydov ${ }^{2}$, P. Poolcharuansin' ${ }^{1}$, T. Vilaithong ${ }^{1}$, I G Brown ${ }^{3}$. \\ ${ }^{1)}$ Department of Physics, Faculty of Science, \\ Chiang Mai University, Chiang Mai 50200 Thailand. \\ ${ }^{2)}$ Institude for Science and Technology Research and Development, \\ Chiang Mai University, Chiang Mai 50200, Thailand. \\ ${ }^{3)}$ Lawrence Berkeley National Laboratory, University of California, Berkeley CA 94720, USA
}

March 1, 2001

This work was supported by National Metal and Materials Technology center and the Thailand Research Fund through the U.S. Department of Energy, under Contract No. DE-AC03$76 \mathrm{SF} 00098$. 


\title{
Optical Emission Spectra of a Copper Plasma Produced by a Metal Vapor
}

\section{Vacuum Arc Plasma Source}

\author{
B. Yotsombat*a , S. Davydov ${ }^{\mathrm{b}}$, P. Poolcharuansin ${ }^{\mathrm{a}}$, T. Vilaithong ${ }^{\mathrm{a}}$, I G Brown ${ }^{\mathrm{c}}$. \\ ${ }^{a}$ Department of Physics, Faculty of Science, Chiang Mai University, Chiang Mai 50200
}

\section{Thailand.}

${ }^{b}$ Institude for Science and Technology Research and Development, Chiang Mai University, Chiang Mai 50200, Thailand.

${ }^{c}$ Lawrence Berkeley National Laboratory, University of California, Berkeley CA 94720, USA

\begin{abstract}
Optical emission spectroscopy in the range from 200 to $800 \mathrm{~nm}$ was applied for investigation of the copper plasma produced by a metal vapor vacuum arc plasma source. The experiments were conducted for the cases when the plasma was guided by straight and $\Omega$ shaped curved solenoids as well as without solenoids and, also, for different vacuum conditions. It was found that, besides singly and doubly charged ions, a relatively high concentration of excited neutral copper atoms was present in the plasma. The relative fraction of excited atoms was much higher in the region close to the cathode surface than in the plasma column inside the solenoid. The concentration of excited neutral, singly-and doubly-ionized atoms increased proportionally when the arc current was increased to 400 A. Some weak lines were attributed to more highly ionized copper species and impurities in the cathode material.
\end{abstract}

Key words: metal vapor vacuum arc plasma source; metal plasma; optical emission spectroscopy

*Corresponding Author, banchoby@hotmail.com 


\section{Introduction}

There has been growing interest in the use of plasma and ion sources based on pulsed metal vapor vacuum arc discharges for direct ion implantation (II) and for plasma immersion ion implantation and deposition (PIIID) technologies [1-4]. An important feature of the vacuum arc discharge is that it can produce a broad spectrum of ion charge states (typically from $1^{+}$to $5^{+}$), as well as neutral atoms and "macroparticles"[1]. The plasma composition depends not only on the cathode material but also on many experimental parameters. In the case of direct ion implantation with a vacuum arc ion source [1-3], the charge-to-mass ratio of the ion components which are extracted from the plasma plume and then accelerated up a few tens of $\mathrm{keV}$ can be determined with the help of a time-of-flight charge state diagnostic [4,5]. In the case of PIIID applications, the ions in the plasma which streams away from the cathode surface are not extracted but guided for a relatively long distance inside a 90-degree or Sshaped curved solenoid before the plasma reaches the sample [2]. Different configurations of magnetic field have been explored for the purpose of increasing the efficiency of plasma transport and to reduce the concentration of neutral particles and macroparticles, which are not acceptable for many applications. The investigation of the plasma composition in this case by time-of-flight or mass spectrometry technique is difficult, especially when compound cathode materials or hybrid systems of metal and gaseous arc discharges are used. Furthermore, the time-of-flight technique does not provide information concerning the fraction of neutral atoms or molecules in the plasma. Optical Emission Spectroscopy (OES) and Laser-Induced Fluorescence (LIF) techniques have been used for the investigation of vacuum arc discharges [6,7]. However, in [6] copper vacuum arc spectra were recorded for the visible region only and for the case of no magnetic ducts for plasma transport. The LIF experiments discussed in [7] were carried out for a very low arc current, lower than typically used for vacuum arc plasma production for II and PIIID techniques. 
In this paper we describe our OES studies in the range $200-800 \mathrm{~nm}$ of the composition of excited neutral and ionized copper species in the plasma produced by our vacuum arc plasma source, especially for the cases when the plasma is guided by straight and by curved magnetic ducts, essentially needed for PIIID applications.

\section{Experiment}

The experimental set-up (plasma source, arc and plasma ion current measurement system) has been described elsewhere [8]. An $8 \mathrm{~mm}$ diameter copper cathode was surrounded by a pyrex tube of $1 \mathrm{~mm}$ wall thickness, in turn surrounded by a ring-shaped trigger electrode made from stainless steel. An aluminum tube with inner diameter of $30 \mathrm{~mm}$ served as anode. Teflon was used as high-voltage insulator and sealing material between the cathode, anode and trigger feedthroughs. The source housing was connected to a $70 \mathrm{~cm}$ diameter by $55 \mathrm{~cm}$ high cylindrical vacuum chamber with many viewports. Normally, the operating pressure in the chamber was normally about $8 \times 10^{-6}$ Torr. In some OES experiments we operated the chamber at a higher gas pressure of up to $10^{-5}$ Torr.

We measured the optical spectra of the copper plasma for various experimental geometries. The first geometry used a direct line-of-sight path from the cathode surface to the optical detector, without any solenoid. In other geometries, the emission spectra were detected using straight and omega-shaped solenoids. The spectra could be monitored along the straight solenoid axis and in the off-axis direction as shown in figure 1(a). Also, it was possible to examine the plasma emission from the end part of the curved solenoid when a shielding screen was used to block the intense emission from the cathode-anode region (figure 
the arc current itself. The arc current was supplied by an LC line (1.4 ohms impedance) which could be charged to a voltage of up to $800 \mathrm{~V}$. This line produced arc current of up to $400 \mathrm{~A}$ with pulse duration about $0.6 \mathrm{~ms}$. A high voltage $(\sim 10 \mathrm{kV})$ surface discharge across the pyrex tube was utilized to trigger the main vacuum arc discharge. A moveable $25 \mathrm{~mm}$ diameter stainless steel flat probe, biased at $-35 \mathrm{~V}$, was used to measure the plasma ion saturation current as a function of arc current.

The OES measurement system included an Ocean Optics S2000 Fiber Optic Spectrometer [9], a 2-meter length fiber optic cable with UV collimating lens (model 74-UVHT), $40 \mathrm{~mm}$ diameter quartz windows, and an additional quartz lens with $30 \mathrm{~mm}$ focal length placed close to the windows. We could obtain a spectral resolution of $1.3 \mathrm{~nm}$ and an accuracy of $0.3 \mathrm{~nm}$ when the system was interfaced to our PC [10]. An integration time of 2 seconds was used to record the plasma emission spectra in single-pulse mode operation.

\section{Results and Discussion}

In table I we list wavelengths of high and low intensity spectral lines (except very weak and overlapping lines) which were observed in the emission of our plasma source in the range from 200 to $800 \mathrm{~nm}$. Table I also shows the excited neutral and ionized species identified using data from references $[11,12]$. It can be seen from the Table that most of the lines belong to singly ionized atoms, with fewer lines belonging to neutral and doubly-ionized atoms. There are also many low intensity lines which could not be identified. A test was conducted to verify that these lines were not caused by extraneous light entering the spectrometer. These lines could originate from the more highly ionized copper species. Contribution to the emission spectra by the residual gas, vapor of Teflon used in the construction of the source housing, and cathode impurity material cannot be ruled out. On the other hand, the 
spectra observed did not differ when the source was operating (for a short time) at a repetition rate of up to 5 pps (200 A arc current) without water-cooling of the cathode and anode flanges.

Figure 2 shows the spectra recorded for the following cases: (a) - when the straight solenoid was used and the radiation was collected in the direction of the solenoid axis (optical fiber sensor position 1); (b) - when the radiation was collected in the off-solenoid-axis direction (position 2); and (c) - when an $\Omega$-shaped curved solenoid was used (position 3). The spectral intensity distribution has been normalized to the peak intensity of the line at $324.7 \mathrm{~nm}$ (Cu I).

It should be noted that in case (a) the emission was detected simultaneously from the low intensity plasma column inside the solenoid and from the high intensity plasma plume close to the cathode surface. The spectrum recorded from the plasma plume emission in the cathode-anode region without the solenoid (which is not shown separately in figure 2) was almost identical to that of case (a). This implied that the plasma column inside the solenoid is optically transparent.

By comparing the various spectra in figure 2 we can see that in case (a) two intense UV lines (324.7 and $327.4 \mathrm{~nm}$ ), belonging to neutral atoms, predominate. These resonance lines with high transition probabilities $\left(\sim 10^{8} s^{-1}\right)$ correspond to the energy levels $2 P_{3 / 2}(3.82$ $\mathrm{eV})$ and $2 P_{1 / 2}(3.786 \mathrm{eV})[11,12]$. Also, in the visible range the intensity of the $510.6 \mathrm{~nm}$ (laser transition [12]) atomic line is much higher than that of the ion lines (for instance, $527 \mathrm{~nm}$ $\mathrm{CuII} / \mathrm{III}, 607.7 \mathrm{~nm} \mathrm{CuII}$ and $655.8 \mathrm{~nm} \mathrm{CuII})$. In case (b) the relative intensities of the lines belonging to ion excited energy levels increase, and in the third case (c) the ion lines predominate. 
It is often supposed that the metal vacuum arc plasma is fully ionized due to the high electron density and temperature in the region close to the cathode surface (about 100 micrometers from the cathode spots) [4]. Figure 2 shows that some concentration of excited neutral atoms exists in the plasma even in the region close to the exit of the $\Omega$-shaped curved solenoid (whereas neutrals are not confined by the magnetic field). The formation of excited neutral atoms in this region can be attributed to electron-ion recombination processes because the plasma remains highly ionized inside the solenoid and is cooled by adiabatic expansion in vacuum. In the region close to the cathode surface, direct electron impact excitation is probably the main process for the formation of excited neutral atoms and, consequently, their relative fraction here is higher in this case.

In figure 3(a) the intensities of some lines corresponding to neutral and ionized species are plotted as a function of arc discharge current. The radiation was collected along the straight solenoid axis. The values of the ion saturation current density as a function of arc current (with and without the solenoid) are given in figure 3(b), the probe was located approximately $30 \mathrm{~mm}$ from the cathode surface (without the solenoid) and at the same distance from the solenoid exit. It can be seen that without the solenoid the ion current density is proportional to the arc current. A small non-linearity occurs when the solenoid is used. It should be noted that figure 3(b)shows the behavior of the integrated ion current of all ion charge states while figure 3(a) provides information concerning the behavior of the population density of certain excited atomic and ionic energy levels. It can be seen that, except for the $655.8 \mathrm{~nm} \mathrm{Cu}$ II line, all other lines show a linear dependence of intensity versus arc current. Also, a linear dependence of the intensities was observed for many other lines of different $\mathrm{Cu}$ species not shown in figure 3(a). This shows (at least in the range of arc current up to $400 \mathrm{~A}$ ) that atoms and ions are mainly excited by electron impact directly from the ground state. The nonlinear dependence of the $655.8 \mathrm{~nm}$ line is worth noting here. The initial level associated 
states as well as by an increase in transitions from higher energy levels. Also, stimulated emission phenomena cannot be ruled out.

To test whether the unidentified lines in the spectra were caused by the presence of residual gas in the vacuum chamber (air or oil vapor from the diffusion pump system), spectra were monitored for the cases when the base pressure prior to the arc discharge was about $10^{-4}$ Torr (figure 4(a)), and when the efficiency of the cold trap of the diffusion pump was reduced (figure 4(b)). It is known that oil molecules from the oil pumping system can be a source of carbon atoms for a metal-carbon composite coatings [13]. As one can see, the spectra in figure 4 differ from those shown in figure 2. Besides copper species lines, there are many new lines. In the first case (with high air pressure) a very intense line at $587.8 \mathrm{~nm}$ appears. In the second case, the intensities of copper atom lines drop sharply while some copper ion lines (485.8 and $655.8 \mathrm{~nm}$ ) become very strong. Some new lines have been identified as belonging to the singly ionized atoms of carbon and nitrogen. At the same time, the intensities of the lines listed in table I as unidentified did not increase and in fact some of these lines were not observed in the spectra of figure 4 .

We conclude that the OES technique discussed in this work is sensitive and can be used to monitor the spectra from small plasma regions inside the solenoid by increasing the integration time and collecting over several discharge pulses. The method is useful for analyzing the composition of the plasma directly in front of the treated samples, especially when the sample is biased at negative voltage for ion acceleration towards its surface. In this connection, we point out that by applying vacuum ultraviolet OES and kinetic spectroscopy techniques one could detail the plasma composition produced by vacuum arc plasma sources, because the strongest spectral lines from highly ionized species lie in the VUV range and the fraction of species can vary during the discharge pulse. 


\section{Conclusion}

An Optical emission spectroscopy technique was applied to study the composition of excited and ionized copper species in a metal vapor vacuum arc discharge plasma source. The emission spectra were recorded for the cases when the plasma was guided by straight and by $\Omega$-shaped curved solenoids, and when viewed directly from the cathode-anode region without any solenoid. It was found that besides singly and doubly charged ions, a relatively high concentration of excited neutral copper atoms was present in the plasma. Some lines were attributed to more highly ionized copper species or to cathode impurity material. The relative fraction of excited neutral atoms was found to be much higher in the region close to the cathode surface than in the plasma column inside the solenoids. The concentration of excited neutral, singly, and doubly ionized atoms increased proportionally when the arc current was increased.

\section{Acknowledgment}

The authors would like to thank M. Rhodes for his help in the operating of the optical spectrometer and P. Pramukkul for her technical help. This work was supported by the National Metal and Materials Technology Center and the Thailand Research Fund. 


\section{$\underline{\text { References }}$}

[1] Brown I G 1994 Rev. Sci. Instrum. 65(10) 3061-81.

[2] Huixing Z, Fengsheng Z, Qiang L, Xiaoji Z, Chengzhou J 1994 Surf. and Coatings Tech. $66345-9$.

[3] Oks E M, Anders A, Brown I G, Dickinson M R, MacGill R A 1996 IEEE Trans. Plasma Sci. 24(3) 1174-83.

[4] Anders A 1997 Surf. and Coatings Tech. 93 158-67.

[5] Brown I G, Galvin J E, MacGilll R A, Wright R T 1987 Rev. Sci. Instrum. 58(9) 158992.

[6] Anders A, Anders S 1991 J.Phys. D: Appl. Phys. 24 1986-1992.

[7] Koyama K, Nakayama Y, Ohi T, Horinouchi K, Sasao H. 1997 IEEE Trans. On Plasma Sci. 25(4) 598-602.

[8] Davydov S, Yotsombat B, Yu LD, Pramukkul P, Charoenukul R, Vilaithong T 2000 Surf. and Coatings Tech. 131 34-43.

[9] Trademark of Ocean Optics. Inc.,Dunedin, FL34698.

[10] Rhodes M W, Wanwilairat S, Vilaithong T, Hoffmann W 2000 Rev. Sci. Instrum. 71 (5)2053-2057.

[11] Lide D R 1994 CRC Handbook of Chemistry and Physics.

[12] Little C E 1999 Metal Vapour Lasers.

[13] Uglov V V, Kuleshov A K, Rusalsky D P, Onate J I, Yang Si-Ze. 2000 Surf. and Coatings Tech. 128-129 150-155. 


\section{Figure Captions}

Figure 1 Schematic diagrams of the experimental set-up and measurement systems.

Figure 2 Spectra of the plasma emission corresponding to the cases when the radiation was collected from the on-axis (a), and off-axis (b), directions of the straight solenoid, and when the $\Omega$-shaped curved solenoid was used (c). Arc current was 300 A.

Figure 3 Intensities of the spectral lines (a), and values of the saturated ion current density (b), as a function of arc current. The optical fiber sensor is in position 1.

Figure 4. Spectra of the plasma emission (with the straight solenoid) for the cases when air (a), and oil vapor (b), pressures were increased up to $10^{-4}$ Torr. The optical fiber sensor is in position 2 . 